\title{
A new factor theorem on absolute Riesz summability
}

\author{
Hüseyin Bor
}




\title{
A NEW FACTOR THEOREM ON ABSOLUTE RIESZ SUMMABILITY
}

\author{
HÜSEYIN BOR
}

Received 06 February, 2014

\begin{abstract}
In this paper, a known theorem dealing with an application of quasi power increasing sequences has extended by using a new class of increasing sequences instead of a quasi- $\sigma$-power increasing sequence. This theorem also includes some new and known results.
\end{abstract}

2010 Mathematics Subject Classification: 26D15; 40D15; 40F05; 40G99; 46A45

Keywords: Riesz mean, absolute summability, infinite series, sequence spaces, power increasing sequences, Hölder inequality, Minkowski inequality

\section{INTRODUCTION}

A positive sequence $X=\left(X_{n}\right)$ is said to be a quasi- $(\sigma, \gamma)$-power increasing sequence if there exists a constant $K=K(X,(\sigma, \gamma)) \geq 1$ such that $K n^{\sigma}(\log n)^{\gamma} X n \geq$ $m^{\sigma}(\log m)^{\gamma} X m$ for all $n \geq m \geq 1$, where $\gamma \geq 0$ and $0<\sigma<1$ (see [14]). If we take $\gamma=0$, then we get a quasi- $\sigma$-power increasing sequence (see [13]). A positive sequence $\left(b_{n}\right)$ is said to be an almost increasing sequence if there exists a positive increasing sequence $\left(c_{n}\right)$ and two positive constants $\mathrm{M}$ and $\mathrm{N}$ such that $M c_{n} \leq b_{n} \leq N c_{n}$ (see [1]). A sequence $\left(\lambda_{n}\right)$ is said to be of bounded variation, denoted by $\left(\lambda_{n}\right) \in \mathscr{B} \mathcal{V}$, if $\sum_{n=1}^{\infty}\left|\Delta \lambda_{n}\right|=\sum_{n=1}^{\infty}\left|\lambda_{n}-\lambda_{n+1}\right|<\infty$. Let $\sum a_{n}$ be a given infinite series with the sequence of partial sums $\left(s_{n}\right)$. We denote by $t_{n}^{\alpha}$ the $n$th Cesàro mean of order $\alpha$, with $\alpha>-1$, of the sequences $\left(n a_{n}\right)$, that is (see[9])

$$
t_{n}^{\alpha}=\frac{1}{A_{n}^{\alpha}} \sum_{v=1}^{n} A_{n-v}^{\alpha-1} v a_{v}, \quad\left(t_{n}{ }^{1}=t_{n}\right)
$$

where

$$
A_{n}^{\alpha}=\frac{(\alpha+1)(\alpha+2) \ldots(\alpha+n)}{n !}=O\left(n^{\alpha}\right), \quad A_{-n}^{\alpha}=0 \quad \text { for } \quad n>0 .
$$

A series $\sum a_{n}$ is said to be summable $|C, \alpha ; \delta|_{k}, k \geq 1$ and $\delta \geq 0$, if (see [11])

$$
\sum_{n=1}^{\infty} n^{\delta k-1}\left|t_{n}^{\alpha}\right|^{k}<\infty .
$$


If we set $\delta=0$, then we get $|C, \alpha|_{k}$ summablility (see [10]).

Let $\left(p_{n}\right)$ be a sequence of positive numbers such that

$$
P_{n}=\sum_{v=0}^{n} p_{v} \rightarrow \infty \quad \text { as } \quad n \rightarrow \infty, \quad\left(P_{-i}=p_{-i}=0, i \geq 1\right)
$$

The sequence-to-sequence transformation

$$
w_{n}=\frac{1}{P_{n}} \sum_{v=0}^{n} p_{v} s_{v}
$$

defines the sequence $\left(w_{n}\right)$ of the Riesz mean or simply the $\left(\bar{N}, p_{n}\right)$ mean of the sequence $\left(s_{n}\right)$, generated by the sequence of coefficients $\left(p_{n}\right)$ (see [12]). The series $\sum a_{n}$ is said to be summable $\left|\bar{N}, p_{n} ; \delta\right|_{k}, k \geq 1$ and $\delta \geq 0$, if (see [4])

$$
\sum_{n=1}^{\infty}\left(P_{n} / p_{n}\right)^{\delta k+k-1}\left|w_{n}-w_{n-1}\right|^{k}<\infty .
$$

If we set $\delta=0$, then we obtain $\left|\bar{N}, p_{n}\right|_{k}$ summability (see [2]). In the special case $p_{n}=1$ for all values of $\mathrm{n}$, the $\left|\bar{N}, p_{n} ; \delta\right|_{k}$ summability is the same as $|C, 1 ; \delta|_{k}$ summability. Also if we take $\delta=0$ and $k=1$, then we get $\left|\bar{N}, p_{n}\right|$ summability.

\section{THE KNOWN RESULT}

The following theorem is known dealing with the absolute Riesz summability factors of infinite series.

Theorem 1 ([5]). Let $\left(X_{n}\right)$ be a quasi- $\sigma$-power increasing sequence for some $\sigma$ $(0<\sigma<1)$ and let there be sequences $\left(\beta_{n}\right)$ and $\left(\lambda_{n}\right)$ such that

$$
\begin{gathered}
\left|\Delta \lambda_{n}\right| \leq \beta_{n}, \\
\beta_{n} \rightarrow 0 \text { as } n \rightarrow \infty, \\
\sum_{n=1}^{\infty} n\left|\Delta \beta_{n}\right| X_{n}<\infty, \\
\left|\lambda_{n}\right| X_{n}=O(1) .
\end{gathered}
$$

If $\left(p_{n}\right)$ is a sequence such that

$$
\begin{aligned}
P_{n} & =O\left(n p_{n}\right), \\
P_{n} \Delta p_{n} & =O\left(p_{n} p_{n+1}\right), \\
\sum_{v=1}^{n}\left(\frac{P_{v}}{p_{v}}\right)^{\delta k} \frac{\left|t_{v}\right|^{k}}{v} & =O\left(X_{n}\right) \text { as } n \rightarrow \infty,
\end{aligned}
$$




$$
\sum_{n=v+1}^{m+1}\left(\frac{P_{n}}{p_{n}}\right)^{\delta k-1} \frac{1}{P_{n-1}}=O\left(\left(\frac{P_{v}}{p_{v}}\right)^{\delta k} \frac{1}{P_{v}}\right) \quad \text { as } \quad m \rightarrow \infty,
$$

then the series $\sum_{n=1}^{\infty} a_{n} \frac{P_{n} \lambda_{n}}{n p_{n}}$ is summable $\left|\bar{N}, p_{n} ; \delta\right|_{k}, k \geq 1$ and $0 \leq \delta<1 / k$.

Remark 1. In the hypothesis of Theorem 1, we have added the condition " $\left(\lambda_{n}\right) \in$ $B \mathcal{V}^{\prime \prime}$.

\section{THE MAIN RESULT}

The aim of this paper is to extend Theorem 1 by using a quasi- $(\sigma, \gamma)$-power increasing sequence instead of quasi- $\sigma$-power increasing sequence. Now we shall prove the following theorem.

Theorem 2. Let $\left(\lambda_{n}\right) \in \mathscr{B V}$ and let $\left(X_{n}\right)$ be a quasi- $(\sigma, \gamma)$-power increasing sequence. If all conditions of Theorem 1 are satisfied, then the series $\sum_{n=1}^{\infty} a_{n} \frac{P_{n} \lambda_{n}}{n p_{n}}$ is summable $\left|\bar{N}, p_{n} ; \delta\right|_{k}, k \geq 1$, and $0 \leq \delta<1 / k$.

Remark 2. If we set $\gamma=0$, then we get Theorem 1. If we take $\delta=0$, then we get a new result dealing with $\left|\bar{N}, p_{n}\right|_{k}$ summability factors. If we take $p_{n}=1$ for all values of $\mathrm{n}$, then we get a new result dealing with $|C, 1 ; \delta|_{k}$ summability factors. Also, if we take $\gamma=0$ and $\delta=0$, the we get the known result (see [6]). Finally, if we take $\left(X_{n}\right)$ as an almost increasing sequence, then we get the known result which was published in [7].

We need the following lemmas for the proof of the theorem.

Lemma 1 ([8]). Except for the condition $\left(\lambda_{n}\right) \in \mathscr{B V}$, under the conditions on $\left(X_{n}\right),\left(\beta_{n}\right)$ and $\left(\lambda_{n}\right)$ as expressed in the statement of the theorem, we have the following;

$$
\begin{aligned}
& \sum_{n=1}^{\infty} \beta_{n} X_{n}<\infty, \\
& n X_{n} \beta_{n}=O(1) .
\end{aligned}
$$

Lemma 2 ([3]). If the conditions (2.5) and (2.6) are satisfied, then we have

$$
\Delta\left(\frac{P_{n}}{n^{2} p_{n}}\right)=O\left(\frac{1}{n^{2}}\right)
$$

\section{Proof of Theorem 2}

Let $\left(T_{n}\right)$ be the sequence of $\left(\bar{N}, p_{n}\right)$ mean of the series $\sum_{n=1}^{\infty} \frac{a_{n} P_{n} \lambda_{n}}{n p_{n}}$. Then, by definition, we have

$$
T_{n}=\frac{1}{P_{n}} \sum_{v=1}^{n} p_{v} \sum_{r=1}^{v} \frac{a_{r} P_{r} \lambda_{r}}{r p_{r}}=\frac{1}{P_{n}} \sum_{v=1}^{n}\left(P_{n}-P_{v-1}\right) \frac{a_{v} P_{v} \lambda_{v}}{v p_{v}} .
$$


Then, for $n \geq 1$ we obtain that

$$
T_{n}-T_{n-1}=\frac{p_{n}}{P_{n} P_{n-1}} \sum_{v=1}^{n} \frac{P_{v-1} P_{v} a_{v} v \lambda_{v}}{v^{2} p_{v}} .
$$

Using Abel's transformation, we get that

$$
\begin{aligned}
T_{n}-T_{n-1}= & \frac{p_{n}}{P_{n} P_{n-1}} \sum_{v=1}^{n-1} \Delta\left(\frac{P_{v-1} P_{v} \lambda_{v}}{v^{2} p_{v}}\right) \sum_{r=1}^{v} r a_{r}+\frac{\lambda_{n}}{n^{2}} \sum_{v=1}^{n} v a_{v} \\
= & \frac{p_{n}}{P_{n} P_{n-1}} \sum_{v=1}^{n-1} \frac{P_{v}}{p_{v}}(v+1) t_{v} p_{v} \frac{\lambda_{v}}{v^{2}} \\
& +\frac{p_{n}}{P_{n} P_{n-1}} \sum_{v=1}^{n-1} P_{v} P_{v} \Delta \lambda_{v}(v+1) \frac{t_{v}}{v^{2} p_{v}} \\
& -\frac{p_{n}}{P_{n} P_{n-1}} \sum_{v=1}^{n-1} P_{v} \lambda_{v+1}(v+1) t_{v} \Delta\left(P_{v} / v^{2} p_{v}\right) \\
& +\lambda_{n} t_{n}(n+1) / n^{2}=T_{n, 1}+T_{n, 2}+T_{n, 3}+T_{n, 4} .
\end{aligned}
$$

To complete the proof of the theorem by Minkowski's inequality, it is sufficient to show that

$$
\sum_{n=1}^{\infty}\left(\frac{P_{n}}{p_{n}}\right)^{\delta k+k-1}\left|T_{n, r}\right|^{k}<\infty, \quad \text { for } \quad r=1,2,3,4 .
$$

Now, applying Hölder's inequality, we have that

$$
\begin{aligned}
& \sum_{n=2}^{m+1}\left(\frac{P_{n}}{p_{n}}\right)^{\delta k+k-1}\left|T_{n, 1}\right|^{k} \\
& =O(1) \sum_{n=2}^{m+1}\left(\frac{P_{n}}{p_{n}}\right)^{\delta k-1} \frac{1}{P_{n-1}^{k}}\left\{\sum_{v=1}^{n-1} \frac{P_{v}}{p_{v}} p_{v}\left|t_{v}\right|\left|\lambda_{v}\right| \frac{1}{v}\right\}^{k} \\
& =O(1) \sum_{n=2}^{m+1}\left(\frac{P_{n}}{p_{n}}\right)^{\delta k-1} \frac{1}{P_{n-1}} \sum_{v=1}^{n-1}\left(\frac{P_{v}}{p_{v}}\right)^{k} p_{v}\left|t_{v}\right|^{k}\left|\lambda_{v}\right|^{k} \frac{1}{v^{k}} \times\left\{\frac{1}{P_{n-1}} \sum_{v=1}^{n-1} p_{v}\right\}^{k-1} \\
& =O(1) \sum_{v=1}^{m}\left(\frac{P_{v}}{p_{v}}\right)^{k} p_{v}\left|t_{v}\right|^{k}\left|\lambda_{v}\right|^{k} \frac{1}{v^{k}} \sum_{n=v+1}^{m+1}\left(\frac{P_{n}}{p_{n}}\right)^{\delta k-1} \frac{1}{P_{n-1}} \\
& =O(1) \sum_{v=1}^{m}\left(\frac{P_{v}}{p_{v}}\right)^{k}\left|\lambda_{v}\right|^{k-1}\left|\lambda_{v}\right| p_{v}\left|t_{v}\right|^{k} \frac{1}{v^{k}} \frac{1}{P_{v}}\left(\frac{P_{v}}{p_{v}}\right)^{\delta k}
\end{aligned}
$$




$$
\begin{aligned}
& =O(1) \sum_{v=1}^{m}\left(\frac{P_{v}}{p_{v}}\right)^{k-1}\left|\lambda_{v}\right|\left|t_{v}\right|^{k} \frac{1}{v^{k}}\left(\frac{P_{v}}{p_{v}}\right)^{\delta k} \\
& =O(1) \sum_{v=1}^{m}\left(\frac{P_{v}}{p_{v}}\right)^{\delta k} v^{k-1} \frac{1}{v^{k}}\left|\lambda_{v}\right|\left|t_{v}\right|^{k} \\
& =O(1) \sum_{v=1}^{m}\left|\lambda_{v}\right|\left(\frac{P_{v}}{p_{v}}\right)^{\delta k} \frac{\left|t_{v}\right|^{k}}{v} \\
& =O(1) \sum_{v=1}^{m-1} \Delta\left|\lambda_{v}\right| \sum_{r=1}^{v}\left(\frac{P_{r}}{p_{r}}\right)^{\delta k} \frac{\left|t_{r}\right|^{k}}{r}+O(1)\left|\lambda_{m}\right| \sum_{v=1}^{m}\left(\frac{P_{v}}{p_{v}}\right)^{\delta k} \frac{\left|t_{v}\right|^{k}}{v} \\
& =O(1) \sum_{v=1}^{m-1}\left|\Delta \lambda_{v}\right| X_{v}+O(1)\left|\lambda_{m}\right| X_{m} \\
& =O(1) \sum_{v=1}^{m-1} \beta_{v} X_{v}+O(1)\left|\lambda_{m}\right| X_{m}=O(1)
\end{aligned}
$$

as $m \rightarrow \infty$, by virtue of the hypotheses of the theorem and Lemma 1. Now, by using (2.5), we have that

$$
\begin{aligned}
& \sum_{n=2}^{m+1}\left(\frac{P_{n}}{p_{n}}\right)^{\delta k+k-1}\left|T_{n, 2}\right|^{k} \\
& =O(1) \sum_{n=2}^{m+1}\left(\frac{P_{n}}{p_{n}}\right)^{\delta k-1} \frac{1}{P_{n-1}^{k}}\left\{\sum_{v=1}^{n-1} P_{v}\left|\Delta \lambda_{v}\right|\left|t_{v}\right|\right\} \\
& =O(1) \sum_{n=2}^{m+1}\left(\frac{P_{n}}{p_{n}}\right)^{\delta k-1} \frac{1}{P_{n-1}} \sum_{v=1}^{n-1} P_{v}\left|\Delta \lambda_{v}\right|\left|t_{v}\right|^{k} \times\left\{\frac{1}{P_{n-1}} \sum_{v=1}^{n-1} P_{v}\left|\Delta \lambda_{v}\right|\right\}^{k-1} \\
& =O(1) \sum_{v=1}^{m} P_{v} \beta_{v}\left|t_{v}\right|^{k} \sum_{n=v+1}^{m+1}\left(\frac{P_{n}}{p_{n}}\right)^{\delta k-1} \frac{1}{P_{n-1}} \\
& =O(1) \sum_{v=1}^{m}\left(\frac{P_{v}}{p_{v}}\right)^{\delta k} \beta_{v}\left|t_{v}\right|^{k}=O(1) \sum_{v=1}^{m} v \beta_{v}\left(\frac{P_{v}}{p_{v}}\right)^{\delta k} \frac{\left|t_{v}\right|^{k}}{v} \\
& =O(1) \sum_{v=1}^{m-1} \Delta\left(v \beta_{v}\right) \sum_{r=1}^{v}\left(\frac{P_{r}}{p_{r}}\right)^{\delta k} \frac{\left|t_{r}\right|^{k}}{r}+O(1) m \beta_{m} \sum_{v=1}^{m}\left(\frac{P_{v}}{p_{v}}\right)^{\delta k} \frac{\left|t_{v}\right|^{k}}{v} \\
& =O(1) \sum_{v=1}^{m-1} v\left|\Delta \beta_{v}\right| X_{v}+O(1) \sum_{v=1}^{m-1} \beta_{v} X_{v}+O(1) m \beta_{m} X_{m}
\end{aligned}
$$




$$
=O(1) \text { as } \quad m \rightarrow \infty
$$

in view of the hypotheses of the theorem and Lemma 1. By using Lemma 2, as in $T_{n, 1}$, we have that

$$
\begin{aligned}
& \sum_{n=2}^{m+1}\left(\frac{P_{n}}{p_{n}}\right)^{\delta k+k-1}\left|T_{n, 3}\right|^{k} \\
& =O(1) \sum_{n=2}^{m+1}\left(\frac{P_{n}}{p_{n}}\right)^{\delta k-1} \frac{1}{P_{n-1}^{k}}\left\{\sum_{v=1}^{n-1} P_{v}\left|\lambda_{v+1}\right|\left|t_{v}\right| \frac{1}{v} \frac{v+1}{v}\right\}^{k} \\
& =O(1) \sum_{n=2}^{m+1}\left(\frac{P_{n}}{p_{n}}\right)^{\delta k-1} \frac{1}{P_{n-1}^{k}}\left\{\sum_{v=1}^{n-1} \frac{P_{v}}{p_{v}} p_{v}\left|\lambda_{v+1}\right| \frac{1}{v}\left|t_{v}\right|\right\}^{k} \\
& =O(1) \sum_{n=2}^{m+1}\left(\frac{P_{n}}{p_{n}}\right)^{\delta k-1} \frac{1}{P_{n-1}} \sum_{v=1}^{n-1}\left(\frac{P_{v}}{p_{v}}\right)^{k} p_{v} \frac{1}{v^{k}}\left|\lambda_{v+1}\right|^{k}\left|t_{v}\right|^{k} \times\left\{\frac{1}{P_{n-1}} \sum_{v=1}^{n-1} p_{v}\right\}^{k-1} \\
& =O(1) \sum_{v=1}^{m}\left(\frac{P_{v}}{p_{v}}\right)^{k} p_{v} \frac{1}{v^{k}}\left|\lambda_{v+1}\right|^{k-1}\left|\lambda_{v+1}\right|\left|t_{v}\right|^{k} \times \sum_{n=v+1}^{m+1}\left(\frac{P_{n}}{p_{n}}\right)^{\delta k-1} \frac{1}{P_{n-1}} \\
& =O(1) \sum_{v=1}^{m}\left(\frac{P_{v}}{p_{v}}\right)^{k-1} \frac{1}{v^{k}}\left|\lambda_{v+1}\right|\left|t_{v}\right|^{k}\left(\frac{P_{v}}{p_{v}}\right)^{\delta k} \\
& =O(1) \sum_{v=1}^{m}\left(\frac{P_{v}}{p_{v}}\right)^{\delta k} v^{k-1} \frac{1}{v^{k}}\left|\lambda_{v+1}\right|\left|t_{v}\right|^{k} \\
& =O(1) \sum_{v=1}^{m}\left(\frac{P_{v}}{p_{v}}\right)^{\delta k}\left|\lambda_{v+1}\right| \frac{\left|t_{v}\right|^{k}}{v}=O(1) \text { as } m \rightarrow \infty .
\end{aligned}
$$

Finally, as in $T_{n, 1}$, we have that

$$
\begin{aligned}
\sum_{n=1}^{m}\left(\frac{P_{n}}{p_{n}}\right)^{\delta k+k-1}\left|T_{n, 4}\right|^{k} & =O(1) \sum_{n=1}^{m}\left(\frac{P_{n}}{p_{n}}\right)^{\delta k}\left(\frac{P_{n}}{p_{n}}\right)^{k-1}\left(\frac{n+1}{n}\right)^{k} \frac{1}{n^{k}}\left|\lambda_{n}\right|^{k}\left|t_{n}\right|^{k} \\
& =O(1) \sum_{n=1}^{m}\left(\frac{P_{n}}{p_{n}}\right)^{\delta k} n^{k-1} \frac{1}{n^{k}}\left|\lambda_{n}\right|^{k-1}\left|\lambda_{n}\right|\left|t_{n}\right|^{k} \\
& =O(1) \sum_{n=1}^{m}\left|\lambda_{n}\right|\left(\frac{P_{n}}{p_{n}}\right)^{\delta k} \frac{\left|t_{n}\right|^{k}}{n}=O(1) \quad \text { as } \quad m \rightarrow \infty
\end{aligned}
$$

This completes the proof of the theorem. 


\section{ACKNOWLEDGEMENT}

The author expresses his thanks to the referee for his/her valuable comments and suggestions for the improvement of this paper.

\section{REFERENCES}

[1] N. K. Bari and S. B. Stečkin, "Best approximation and differential properties of two conjugate functions," Trudy. Moskov. Mat. Obšč., vol. 5, no. 1, pp. 483-522, 1956.

[2] H. Bor, "On two summability methods," Math. Proc. Camb. Philos Soc, vol. 97, no. 1, pp. 147$149,1985$.

[3] H. Bor, "Absolute summability factors for infinite series," Indian J. Pure Appl. Math., vol. 19, no. 7, pp. 664-671, 1988.

[4] H. Bor, "On local property of $\left|\bar{N}, p_{n} ; \delta\right|_{k}$ summability of factored Fourier series," J. Math. Anal. Appl., vol. 179, no. 2, pp. 646-649, 1993.

[5] H. Bor, "On some new applications of increasing sequences," Advan. Stud. Contemp. Math., vol. 17, no. 2, pp. 231-239, 2008.

[6] H. Bor, "A theorem on the absolute summability factors," Math. Notes, vol. 86, no. 3-4, pp. 463468, 2009.

[7] H. Bor, "On an application of almost increasing sequences," Math. Commun., vol. 15, no. 1, pp. 57-64, 2010.

[8] H. Bor, "A new application of generalized power increasing sequences," Filomat, vol. 26, no. 3, pp. 631-635, 2012.

[9] E. Cesàro, "Sur la multiplication des séries," Bull. Sci. Math., vol. 14, no. 1, pp. 114-120, 1890.

[10] T. M. Flett, "On an extension of absolute summability and some theorems of Littlewood and Paley,” Proc. London Math. Soc., vol. 7, no. 1, pp. 113-141, 1957.

[11] T. M. Flett, "Some more theorems concerning the absolute summability of fourier series," Proc. London Math. Soc., vol. 8, no. 3, pp. 357-387, 1958.

[12] G. H. Hardy, Divergent Series. Oxford: Oxford Univ. Press, 1949.

[13] L. Leindler, "A new application of quasi power increasing sequences," Publ. Math. Debrecen, vol. 58, no. 4, pp. 791-796, 2001.

[14] W. T. Sulaiman, "Extension on absolute summability factors of infinite series," J. Math. Anal. Appl., vol. 322, no. 2, pp. 1224-1230, 2006.

Author's address

\section{Hüseyin Bor}

P. O. Box 121, TR-06502 Bahçelievler, Ankara, Turkey

E-mail address: hbor33@gmail.com 